Projets

de paysage

\section{Projets de paysage}

Revue scientifique sur la conception et l'aménagement de l'espace

$22 \mid 2020$

Forêt et paysage

\title{
Vivre de paysage dans le Morvan ou l'impensé de la gestion forestière morvandelle
}

Experiencing the Landscape in the Morvan or the Implied Management of the Morvan Forest

\section{Damien Marage}

\section{CpenEdition}

\section{Journals}

Édition électronique

URL : http://journals.openedition.org/paysage/8182

DOI : 10.4000/paysage.8182

ISSN : 1969-6124

Éditeur :

École nationale supérieure du paysage de Versailles-Marseille, Institut national des sciences appliquées Centre Val de Loire - École de la nature et du paysage, École nationale supérieure d'architecture et de paysage de Bordeaux, École nationale supérieure d'architecture et de paysage de Lille, Agrocampus Angers

\section{Référence électronique}

Damien Marage, « Vivre de paysage dans le Morvan ou l'impensé de la gestion forestière morvandelle », Projets de paysage [En ligne], 22 | 2020, mis en ligne le 21 juillet 2020, consulté le 24 juillet 2020. URL : http://journals.openedition.org/paysage/8182 ; DOI : https://doi.org/10.4000/ paysage.8182

Ce document a été généré automatiquement le 24 juillet 2020

Projets de paysage 


\title{
Vivre de paysage dans le Morvan ou l'impensé de la gestion forestière morvandelle
}

\author{
Experiencing the Landscape in the Morvan or the Implied Management of the \\ Morvan Forest
}

Damien Marage

Depuis 2016, les chartes des parcs naturels régionaux doivent fixer, de par la loi de reconquête de la biodiversité et des paysages, des objectifs de qualité paysagère. À l'occasion de la révision de la charte du Parc naturel régional du Morvan (PNRM), le paysage en est devenu le fil rouge, les parties prenantes faisant le double constat que considérer l'avenir d'un paysage forestier oblige à prendre en compte de façon systémique les activités qui se déploient sur ce territoire et surtout que le paysage peut être un excellent levier de motivation et de médiation entre acteurs.

2 La prégnance de la problématique forestière est patente dans le Morvan. Les aides du Fonds forestier national et des aides régionales, dans un contexte de déprise agricole, ont conduit depuis 70 ans à un enrésinement conséquent de ce massif. La plupart des peuplements de résineux arrivant à maturité et étant fragilisés par les effets du changement climatique, le problème de leur renouvellement est posé et, avec lui, le modèle de gestion forestière qui s'y rattache. Le sujet suscite un débat public virulent, qui est alimenté par l'augmentation du rythme des coupes rases qui est vécue comme une atteinte à la qualité paysagère par un nombre croissant d'habitants et d'acteurs locaux. L'impact d'ores et déjà spectaculaire du dérèglement climatique sur la santé des forêts via les attaques de scolytes contribue à alimenter ce débat. De plus la monospécificité des plantations joue un rôle dans la propagation du scolyte.

3 Parce que la forêt structure nos paysages, qu'elle est source de multiples usages, parce qu'elle hante nos inconscients collectifs, la forêt invite chacun de nous à poser sur elle des regards pluriels, elle est chose publique (Marage, 2018). Or, les choix de gestion forestière sont systématiquement motivés au nom d'une sacro-sainte durabilité de la 
gestion. Notion quelque peu équivoque, puisqu'on voudrait tout à la fois conserver les forêts, tout en alimentant la filière-bois locale et internationale et répondre aux attentes des habitants du territoire et à une société de loisirs.

4 Le paysage forestier ne peut plus être déconnecté des choix économiques de notre société. Le paysage devient à la fois un patrimoine, un capital dont les acteurs attendent les intérêts, un terroir avec sa production locale. La gestion multifonctionnelle des forêts morvandelles méritait donc d'être questionnée dans cette future charte de parc 2020-2035. Se trouve imbriquée, en creux, la problématique de la spécialisation de ce territoire forestier (Barthod, 2015), les forêts morvandelles étant considérées historiquement comme "nourricières » et aujourd'hui comme " une usine à bois ». Lors de la phase de concertation de la future charte, la qualité paysagère des massifs forestiers a été source d'incompréhensions et a conduit à la rupture du dialogue entre les parties prenantes. Si les paysages du Morvan sont aussi considérés comme une ressource, un patrimoine, qu'ils soient gérés par les populations locales ou accaparés par des intérêts extérieurs, leur fragilité concerne au premier chef les populations locales qui seront les premières à en pâtir en cas de dégradation (Déry, 2012). Le télescopage entre la révision de la charte du Parc et la rédaction du contrat régional forêt-bois de la région Bourgogne-Franche-Comté a provoqué des incompréhensions, a fragilisé, jusqu'à les rompre, les liens entre acteurs.

5 Le paysage ne peut plus être réduit à « la partie d'un pays que la nature présente à un observateur " selon la définition du Petit Robert. Ce couplage sujet-objet propre à la définition occidentale historique du paysage pourrait laisser place à une vision orientale où le paysage dit la corrélation du Haut et du Bas, de l'immobile et du mouvant, de ce qui a forme et de ce qui est sans forme, ou encore de ce qu'on voit et de ce qu'on entend (Jullien, 2014). Dans ce champ de tension instauré par le paysage, le perceptif devient en même temps affectif et de ces formes qui sont aussi des flux se dégage une dimension d'« esprit » qui fait entrer en connivence. Au contraire, et ce sera notre base de travail : il y a « paysage » quand le pays se met en tension; quand ses différents éléments entrent en interaction, s'organisent en polarité et qu'en découle un accroissement manifeste d'intensité. Cette conception du paysage se rapproche de la formulation de Paul Arnould (2003) qui réunit une vision systémique et celle de la géographie culturelle. Dans ce découpage objet-sujet, les paysages forestiers du Morvan offrent trop souvent un spectacle de désolation, de vide induit par les coupes rases. Il faut dépasser cette dichotomie du sujet et de l'objet pour inscrire le paysage dans un «projet ». L'intérêt de cette formalisation est de déboucher sur des hypothèses, sur les hiérarchies et les interactions entre ces systèmes interdépendants.

Par un traitement des attentes culturelles, l'enrichissement et la validation du projet de charte par la population sont appelés à faire vivre le paysage (Brédif et al., 2017). Dans nos sociétés, le territoire et son paysage ne peuvent plus être le fruit d'une décision supérieure, pas plus que la résultante des préconisations des experts, mais le fruit d'un consensus large entre les différents groupes sociaux (Marage, 2018). La production de catégories émergentes d'acteurs à partir d'enceintes de concertation territorialisée permet de les faire travailler autour de ce qui peut être préservé en commun: le paysage n'est plus affaire de " point de vue » ou de « vision du monde», mais du vivre. Ces oppositions ne sont donc pas une fatalité. L'issue à ces antagonismes se trouve, selon nous, dans la construction d'une vision partagée de l'avenir du paysage forestier du Morvan, dans la production d'innovations sociales, source de transitions. Nous 
étayerons, pour cela, nos propos grâce aux nombreux diagnostics, bilans et enquêtes réalisés par ou pour le PNRM, ces dernières années.

\section{La forêt morvandelle : ses peuplements forestiers, leur gestion et la genèse des paysages contemporains}

7 Le Morvan constitue, au cœur de l'ex-région de Bourgogne, une petite montagne centrale qui culmine à $901 \mathrm{~m}$ d'altitude (figure 1). Assises métamorphiques, sédimentaires, volcaniques primaires et socle granitique en composent le substratum. Cette ambiance géochimique acide retentit sur la végétation : les landes sont à callune, fougère aigle et genêt à balais, les prairies sont à trisète commune (Trisetum flavescens), canche flexueuse (Deschampsia flexuosa) et flouve odorante (Anthoxanthum odoratum). Premier relief exposé aux dépressions océaniques, le massif du Morvan reçoit entre 1000 à $1500 \mathrm{~mm}$ de pluie par an (Boulard, 2016). Ce contexte géopédoclimatique est très propice à la végétation forestière.

Les paysages sont dominés pour moitié par les prairies au sein desquelles subsiste un bocage encore dense et par la forêt qui couvre un peu plus de la moitié de ce massif soit 128000 ha. Les milieux forestiers se répartissent en trois ensembles (Rameau, 1985 ; Simonnot 1991): les forêts acidiphiles largement répandues sur des sols acides; les forêts mésoacidiphiles à neutrophiles occupent des sols plus riches, situés en bas de versant ou sur des placages de limon dissimulant le substrat cristallin. Enfin les fonds de vallon hébergent des forêts rivulaires linéaires mésohygrophiles à hygrophiles.

Figure 1. Situation du massif du Morvan

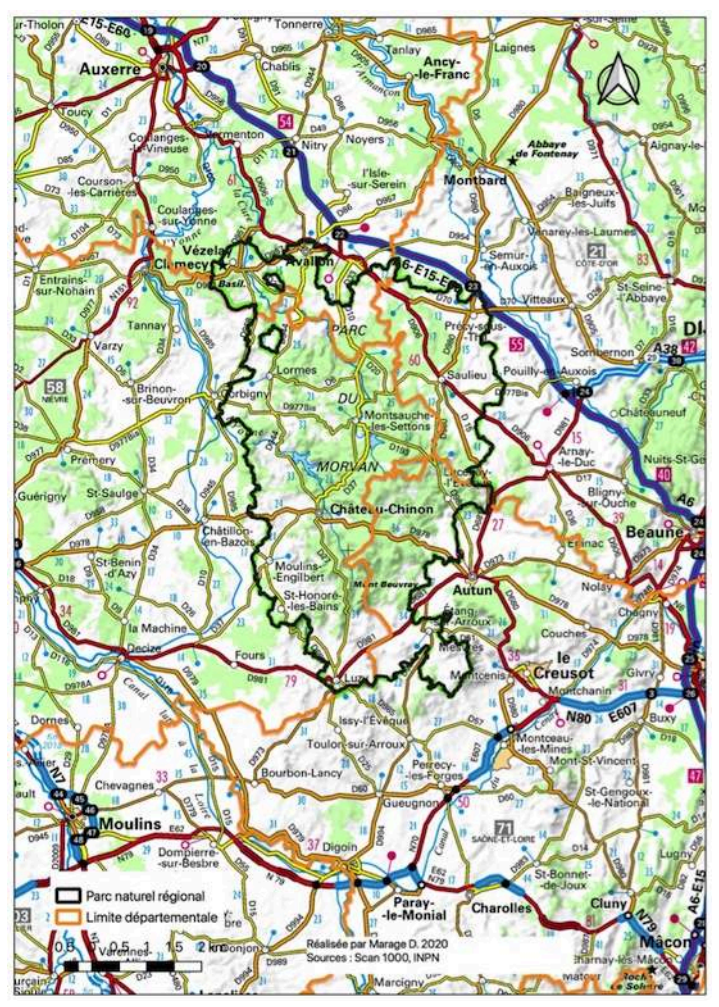

Source : Damien Marage. 


\section{D'un territoire organisé à « nourrir » Paris en bois à une forêt destinée à « nourrir » le marché international en bois}

9 Ce relief hercynien émoussé (figure 1) a donné à cette zone de montagne une double vocation agricole et forestière. Parce que les conditions d'exploitation y sont plus faciles qu'ailleurs et que Paris, ceint de forêts royales inaliénables, ne peut pourvoir à son approvisionnement en bois de feu, le flottage est organisé à partir de 1547 et ne cessera définitivement son activité qu'en 1927. Le port de Clamecy est, dans cette industrie, d'une importance cruciale (figure 2) (Dupont 1995). À l'aube de la Révolution, un million de stères sont délivrés à Paris chaque année. La forêt morvandelle passe alors d'une exploitation domestique et usagère au Moyen Âge à une exploitation industrielle et commerciale à l'époque moderne. C'est une culture de "coupeurs" plutôt que de sylviculteurs, le traitement en taillis fureté ne réclamant pas de grandes connaissances et compétences en sylviculture (Paris, 2011). L'exploitation est de type "minière " même si les auteurs prennent soin d'indiquer "qu'on ne saignait pas la forêt à blanc. » À l'exception de quelques forêts royales ou ecclésiastiques passées dans le giron de l'État lors de la Révolution française (Breuil-Chenue, Haut-Folin, Planoise...), les surfaces forestières sont en net recul à la fin du xixe siècle d'environ $20 \%$ de la superficie du Morvan contre $55 \%$ aujourd'hui (Deconninck, 2011).

Figure 2. Flottage « à bûches perdues » du bois pour Paris aux abords de Clamecy vers 1900

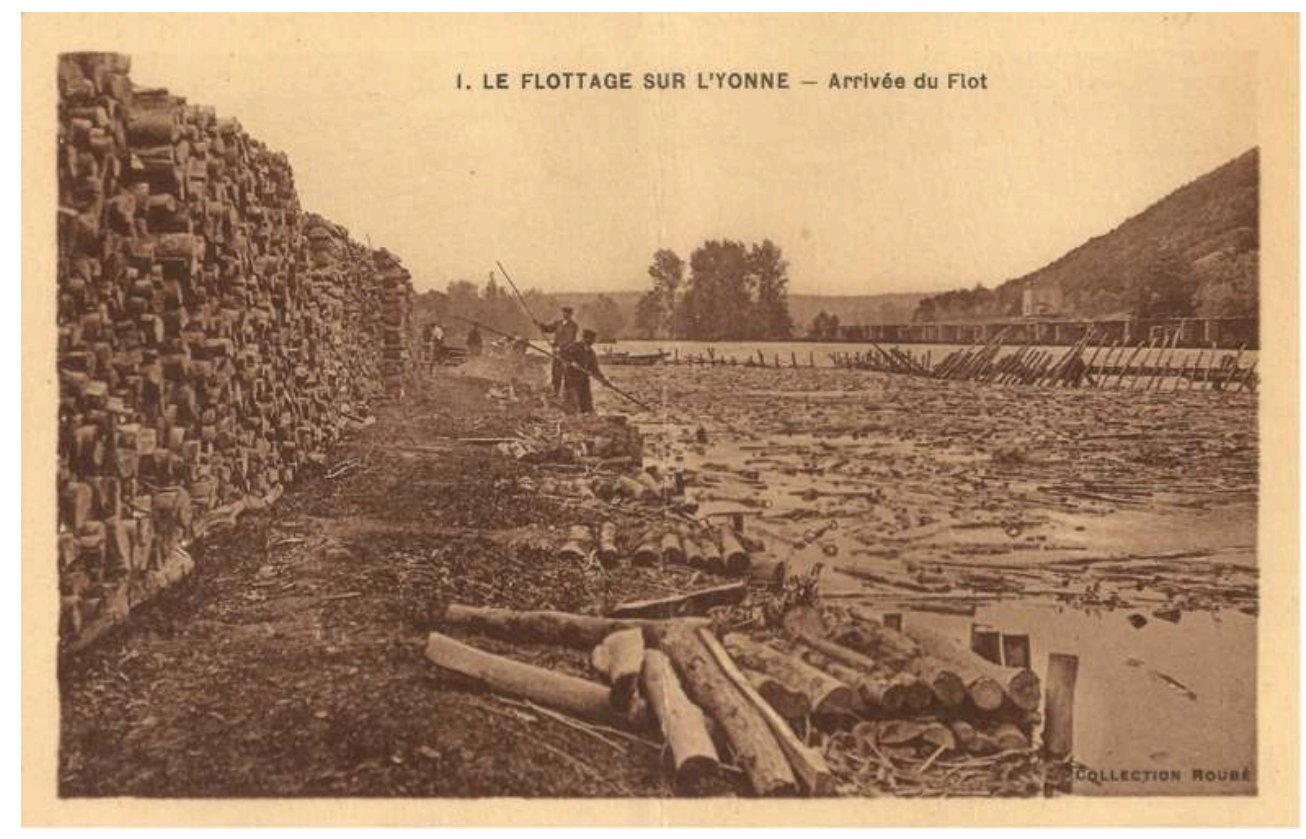

Source : DR.

De 1927 à 1945, les forêts morvandelles sont délaissées. Entre-temps, l'ancienne noblesse du territoire a soit conservé ces domaines forestiers, soit les a vendus à des institutionnels. C'est que la forêt morvandelle appartient à plus de 18000 propriétaires qui en détiennent $85 \%$ (Deconninck, 2011).

11 Au sortir de la Seconde Guerre mondiale, le 30 septembre 1946, le Fonds forestier national (FFN) est créé. Il veut rendre à la France son autonomie en pâte à papier, bois 
d'industrie et bois résineux. À l'instar des Ardennes et du Massif central, les forêts morvandelles, dont les hêtraies et chênaies-hêtraies sortent "épuisées " de quatre siècles d'exploitation forestière intensive, sont transformées en boisements résineux, d'abord en sapins (Abies alba, A. grandis) et épicéa commun (Picea abies) puis majoritairement en douglas (Pseudotsuga menzessii) (Ranger et al., 2011). Les paysages forestiers actuels du Morvan sont donc structurés en mosaïque, véritable patchwork de peuplements feuillus-résineux et mixtes (figure 3).

Figure 3. Mosaïque paysagère depuis une vue aérienne du mont Beuvray

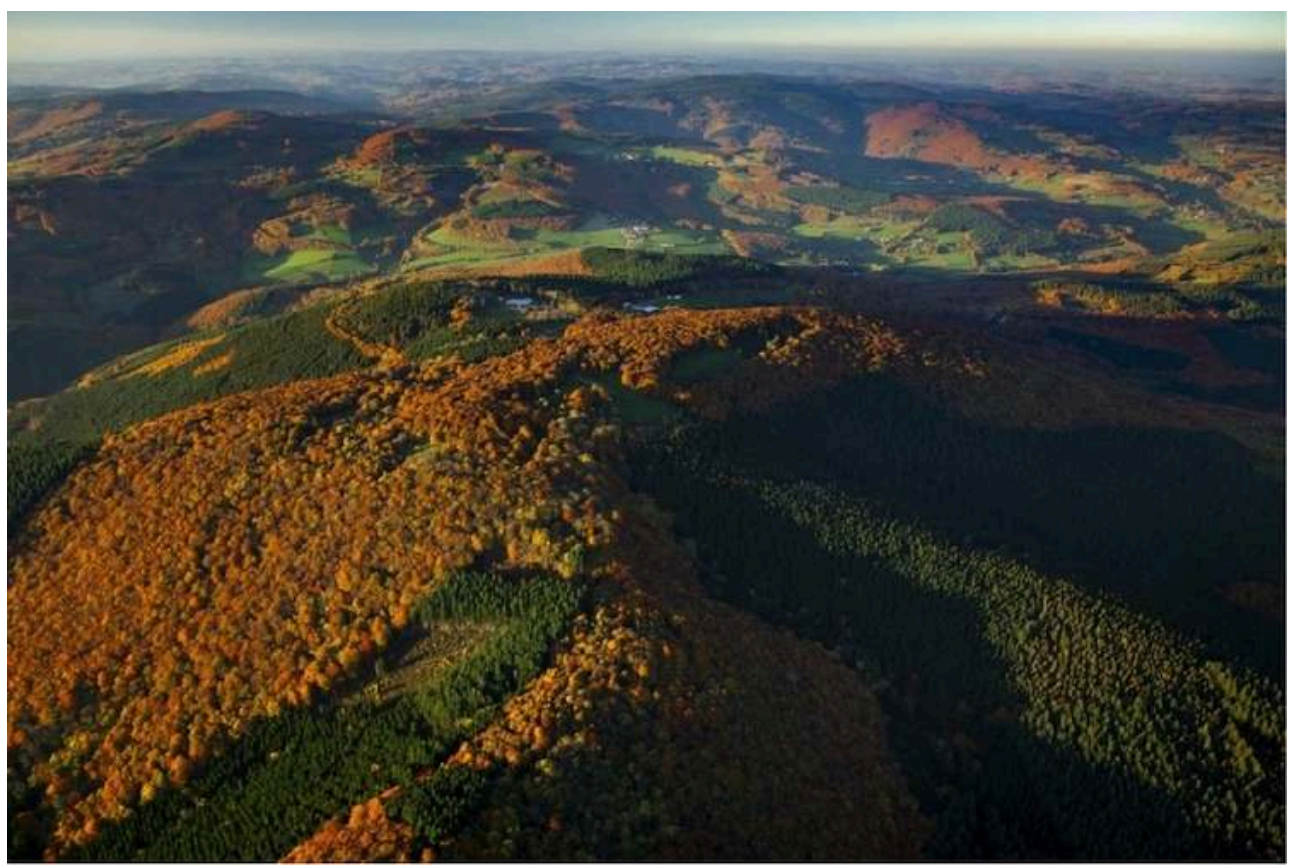

Site de l'ancienne capitale gauloise de Bibracte.

Source : Bourgogne Live, Aurélien Ibanez.

12 Tout comme les plateaux de Millevaches en Limousin, le relief peu marqué du Morvan en fait un territoire propice à la mécanisation forestière. Si l'État a largement financé les plantations résineuses via le FFN, les lourds investissements demandés pour l'acquisition d'abatteuses, de débusqueurs et de porteurs sont financés par la région. Selon une étude de Forêt Cellulose Bois-construction Ameublement (FCBA) (Ruch et Montagny, 2015), 301 machines dont 54 abatteuses, 56 débusqueurs et 90 porteurs ont été recensés pour 148 entreprises de travaux forestiers. L'ex-région Bourgogne était donc la deuxième région française en matière de mécanisation forestière derrière le massif landais.

13 C'est qu'il faut récolter une ressource forestière, principalement constituée de douglas (10 mm3 sur pied). Selon, l'enquête annuelle de la branche "exploitation forestière ", ce sont $500000 \mathrm{~m} 3 /$ an de douglas qui ont été récoltés en 2014. Ces peuplements arrivant à maturité dans la prochaine décennie, selon les études de disponibilité de la ressource forestière, ce sont environ 1,4 million de m3/an de douglas qu'il est prévu de récolter à l'horizon 2030 (Contrat régional forêt-bois de Bourgogne-Franche-Comté, 2019).

14 Pourquoi les forestiers sont-ils aujourd'hui régulièrement interpellés lorsque leurs pratiques de récolte deviennent trop visibles, dans des forêts pourtant plus que 
gérées ? La phase d'exploitation, c'est-à-dire les coupes d'éclaircie, de régénération, définitives, à blanc..., cristallise toutes les tensions.

\section{Un divorce préoccupant : la forêt et le bois}

«bois ». Pour lui :

«[...] la forêt est un objet sympathique et éthique, quelque chose qui mérite d'être défendu ; d'un autre côté, le bois matériau renouvelable est un objet sympathique, susceptible de contribuer à trouver des solutions à des problèmes qui dépassent la seule forêt. Néanmoins, entre les deux la gestion forestière et la récolte du bois sont vues comme "occasionnant des dégâts", sans doute en introduisant l'industrie dans la nature, l'économique dans la contemplation » (Barthod, 2019). notion de conservation, chère au code forestier, quand les engins imposants de type abatteuses ou débusqueurs entrent en forêt, prélèvent les arbres à récolter, créent souvent des ornières dans les chemins et tassent les sols, même si certains engins sont équipés de pneus plus larges. C'est aussi une question de vitesse et de désynchronisation: à la lenteur de croissance des arbres s'opposent les quelques minutes, voire secondes lorsqu'il s'agit de l'abattre.

Comme le souligne Paul Arnould :

«Il est ainsi possible d'affirmer que le paysage forestier a été ignoré par les deux sphères les plus influentes dans l'aménagement, celle des ingénieurs, des techniciens, des économistes et celle des politiques et des juristes » (Arnould, 2003).

Parvenir à renouer le lien entre la forêt, considérée comme un espace naturel, et les usages du bois, matériau de construction, n'est pas qu'un défi de forestiers, mais bien un défi sociétal.

\section{La dimension sociale des paysages forestiers morvandiaux}

Andrée Corvol a démontré comment l'espace forestier a d'abord été perçu, depuis la fin du xixe siècle, comme un milieu naturel par les urbains ; il n'est plus associé à l'idée de travail, à la suite de la dislocation du couple espace forestier-tenure paysanne et de l'apparition d'un nouveau couple espace forestier-volonté citadine (Corvol, 1995). Cette dislocation du couple espace forestier-tenure paysanne s'est réalisée dans le Morvan dans l'entre-deux-guerres, plus tardivement que dans d'autres territoires forestiers.

Depuis plus de 40 ans, les habitants réclament de plus en plus le droit d'être associés à la prise de décision (Hotyat, 1975). De plus en plus de citoyens contestataires refusent les grandes coupes rases et la brutalité de l'évolution des paysages (figure 4). Ils ont des attentes en matière de naturalité, de protection des sols, de diversité des âges et des essences.

21 Sont-ils et peuvent-ils être associés lorsque la forêt privée occupe 50 à $90 \%$ de la surface forestière de certaines communes? Ce qu'ils réclament, sans le savoir, c'est la pratique d'une gestion écosystémique, d'une gestion socio-écosystémique. On pourrait ajouter que les communes ne sont pas non plus consultées sur les travaux de coupe et plan de gestion... 


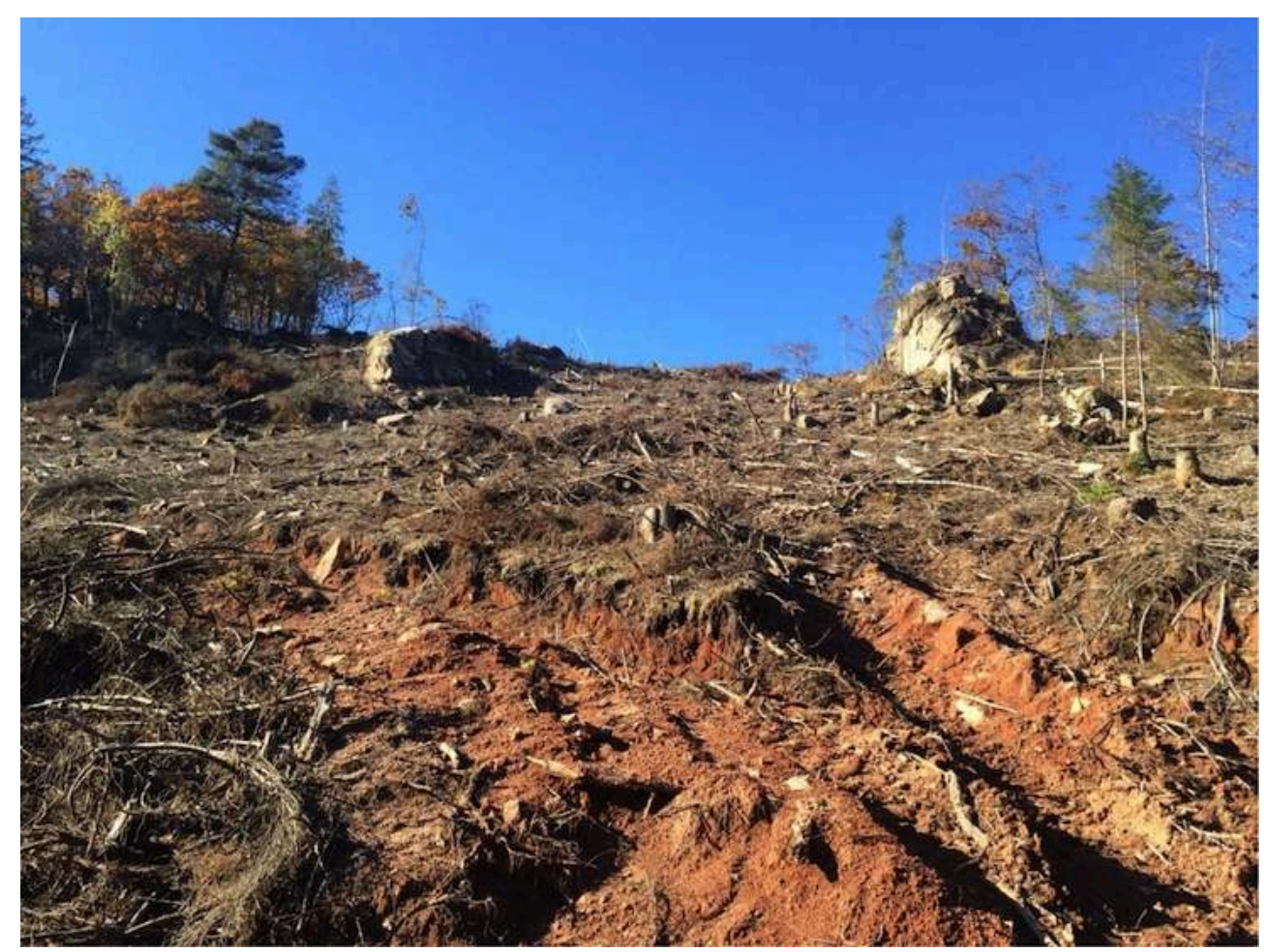

Secteur de Dun-les-places.

Source : Groupement forestier du Chat sauvage.

Doit-on conserver pour cela des forêts multifonctionnelles dans tous les territoires comme nous l'intime le code forestier (Nemoz-Rajot, 1998), ou bien proposer des forêts spécialisées: forêts industrielles à renfort d'intrants, forêts réserves biologiques intégrales, forêts de promenade et de bien-être, forêts de cueillette artisanale...

23 La gestion multifonctionnelle des forêts, prenant en compte à la fois les dimensions économique, sociale et environnementale de leur exploitation, est la plus à même, dans la plupart des cas, de répondre tant aux besoins du marché qu'à ceux des populations locales, tout en faisant bénéficier des services rendus par la forêt à l'environnement. Cette gestion multifonctionnelle convient-elle aux innombrables propriétaires du Morvan?

\section{Diagnostics, audit, concertations, participation : une panoplie d'outils pour apaiser}

Dès la création du PNR du Morvan en 1970, les pratiques de gestion et d'exploitation forestières inspirées des méthodes agronomiques des plus conventionnelles (plantation monospécifique, récolte et reboisement) suscitent des mécontentements et récriminations, comme en témoignent les travaux pionniers de Micheline Hotyat sur le territoire communal de Dun-les-Places (Hotyat, 1975). Ce sont ces tensions pesantes sur le développement territorial que le PNR a à gérer depuis maintenant 50 ans.

En 1994, le préfet de région instaure un groupe de coordination des actions de développement de la forêt morvandelle dont la présidence est confiée à la sous- 
préfecture de Château-Chinon. Fort de belles avancées comme le guide simplifié des stations forestières du Morvan (Oberti, 1997) et surtout le cahier de recommandations paysagères (Breman, 1997), ce groupe réunit l'ensemble des partenaires forestiers et des usagers. Les Orientations régionales forestières de Bourgogne les reprennent à leur compte.

Mais, six ans plus tard, la direction de la Nature et des Paysages, chargée de la politique des PNR, commande un audit stratégique sur le territoire du PNRM. Cet audit fait le constat que la multiplicité des regards génère un sentiment de grand désarroi (Claudez, 2000) : les associations regrettent que des décisions (gestion) ayant des répercussions si importantes pour leur cadre de vie (coupe rase) ne soient pas largement débattues. Les forestiers sont conscients du changement radical et rapide du paysage mais déplorent le décalage entre le paysage réel et le paysage "souhaité dans les têtes ». Ils perçoivent ces récriminations comme injustes, persuadés de l'intérêt général de leurs actions (alimentation de la filière bois). Les investisseurs qui s'appuient sur les aides du FFN craignent une multiplication des contraintes liées à la prise en compte de la qualité des paysages au nom des aspirations sociétales. Ils sont très explicitement dans une gestion monofonctionnelle (production de bois, rentabilité économique, retour sur investissement). Ils demandent des compensations financières s'il faut revenir à une gestion multifonctionnelle. Les acteurs s'inscrivant dans une perspective territoriale ont une vision plus systémique. Ils prônent ce que l'on peut nommer la qualité totale du territoire (Ollagnon, 2006). La légitimité de ces différentes visions du monde n'est pas à remettre en cause.

Un an plus tard, le PNR du Morvan s'empare des chartes forestières de territoires (CFT), instituées par la loi d'orientation forestière (2001) et, en 2005, initie une des premières CFT multithématiques (Riethmuller et al., 2003) avec des actions pilotes diagnostics concernant l'irrégularisation de peuplements résineux ; des itinéraires pour le mélange feuillus-résineux et pour la mise en place d'îlots de vieillissement dans les forêts bénéficiant du régime forestier; la cartographie des problèmes de franchissement de ruisseaux sur dessertes forestières et la mise en place de franchissements permanents ou temporaires et des fiches pratiques pour la prise en compte du paysage dans les opérations sylvicoles.

\section{Emboîtement de chartes, révision de charte et cristallisation des postures}

$\mathrm{Au}$ terme de la 4e CFT (2016-2020), ce sont plus de 5000 brochures éditées, l'organisation de plusieurs séminaires sur les opportunités économiques de la biodiversité forestière (2018) et sur les sols forestiers (2019); ce sont la remise de sylvotrophées depuis 2018, la mise en place d'un marteloscope et d'actions de formation et de sensibilisation auprès des établissements scolaires. C'est un travail conséquent, opiniâtre qui a été réalisé par l'ensemble des acteurs ces quinze dernières années dans le cadre des CFT. Pour les élus du Morvan, un guide du débardage a été élaboré par les services de l'État en 2015 afin de retrouver la maitrise des chemins ruraux et de la voirie communale lors des chantiers d'exploitation forestière.

Mais rien n'y fait, pour les propriétaires forestiers privés, il semble qu'il faille privilégier la sensibilisation et les explications plutôt que la réglementation. En effet, dans la bouche du syndicalisme de la propriété forestière, on entend des sentences des 
plus libertaires comme celles d'un certain mois de mai 1968: "Il est interdit d'interdire. " Depuis plus de 250 ans, c'est le même discours, celui des physiocrates (Saint-Jacob, 1960). On ne peut s'attaquer à la liberté et surtout pas au droit de propriété découlant de l'article 544 du code civil (Ost, 1995). Il faut donc privilégier l'assouplissement du cadre d'emploi des aides financières, notamment sur les essences autorisées, et permettre une meilleure adaptation au changement climatique. Cependant, en matière de préservation du paysage et de conservation de la nature, les parcelles, la propriété forestière sont soumises aux regards des autres, de la société.

Comment, tout en restant dans le cadre actuel législatif, dépasser cette posture, sans la rendre vaine ? L'impact paysager d'une coupe suivie d'enrésinement est en effet quasi immédiat et ne nécessite pas de connaissances spécifiques, ni de protocoles d'évaluation très sophistiqués pour évaluer les conséquences de cette transformation (Bouleau et al., 2016). La mobilisation des associations locales de protection des paysages, des élus, et des habitants était donc plus facile, ainsi que l'imputation des causes. L'administration s'est appliquée à maintenir le débat dans la sphère technique en proposant notamment toute une série de recommandations et de directives visant à une intégration paysagère des opérations sylvicoles comme les Orientations régionales forestières (ORF), puis le Schéma régional de gestion sylvicole (SRGS) mais sans portée réglementaire.

31 Dans ce contexte de tensions récurrentes, une étude sociologique est commandée spécifiquement pour le Morvan par la Direction régionale de l'alimentation, de l'agriculture et de la forêt (DRAAF) de Bourgogne-Franche-Comté dans le cadre de l'élaboration du contrat régional forêt-bois en 2017. Ces travaux s'attachaient à examiner comment la mobilisation supplémentaire de la ressource forestière dans le Morvan pouvait être acceptée localement et quels étaient les moyens de faire converger les points de vue autour d'une approche participative (Haidara, 2018). Les conclusions sont sans appel : la typologie des acteurs et leurs préoccupations n'ont pas changé depuis l'audit de Patrick Claudez de 2000, à l'exception du changement climatique. À ce propos, Roxane Sansilvestri et al. (2020) soulignent que la capacité d'adaptation à long terme de la forêt morvandelle pourrait être remise en question. À l'instar $\mathrm{du}$ modèle forestier landais, cette stratégie à sens unique réduit potentiellement le nombre de trajectoires futures possibles pour le socioécosystème forestier du Morvan. Ainsi, ses acteurs forestiers réduisent actuellement leur capacité d'adaptation en développant uniquement des productions forestières encore bien réparties sur le territoire, mais ne rendent pas cette stratégie socialement acceptable. Les parties prenantes doivent rapidement faire prendre conscience des risques climatiques futurs. Selon Sansilvestri et al. (2019), une gestion multitrajectoire doit être développée pour éviter les pièges de l'«impasse de robustesse » vécue par le massif landais à la suite des tempêtes de 2009.

Ainsi, les futurs documents de gestion devraient s'intéresser à une gestion globale des territoires dans laquelle la forêt n'est finalement qu'une composante de la mosaïque paysagère. Dans les provinces du Québec, cette gestion intégrée est mise en œuvre depuis 2004 (Domon et al., 2004). Dans le PNR des Alpilles, Camille Rivière s'est heurté au même problème: les documents de gestion forestière n'intègrent pas assez les aspects écologiques, ne promeuvent pas toujours des modes de production de bois compatibles avec l'exigence de durabilité dans des aires protégées comme le sont les parcs naturels régionaux (Rivière, 2017). 

en question dans la décennie 2010-2020. Avec l'arrivée à maturité des peuplements de douglas, les incitations et la loi de 2015 instaurant le plan national forêt-bois, sa déclinaison régionale dans un contrat régional forêt-bois à l'heure ou se renouvelle la charte du PNRM, chacun campe sur sa propre vision du monde: les associations militantes prônent la gestion écologique, voire la non-gestion; les gestionnaires, des itinéraires sylvicoles conventionnels; les collectivités sont déconnectées des choix de gestion puisqu'elles n'ont pas de patrimoine forestier à gérer, les habitants sont spectateurs. Face à cette situation complexe, une forme d'atonie dans la concertation et une césure entre ce groupe institutionnalisé et la population, les élus, les associations vont contribuer à affaiblir les efforts entrepris. Serait-ce, comme Pierre Dérioz (2013) le souligne, un enjeu majeur pour les PNR mais une mission impossible que celle du paysage?

Comment dans les documents de gestion forestière retisser le lien entre les entités naturelles et les entités humaines, pour faire monde commun? Comment pour un syndicat mixte de PNR relever le défi de la qualité paysagère dans ces conditions? Estce, finalement, possible de vivre de paysage dans le Morvan?

\section{Vivre de paysage dans le Morvan, à la croisée des chemins}

\section{Un massif forestier, des trajectoires paysagères différentes}

Les forêts morvandelles, comme celles des autres territoires d'ailleurs, sont soumises à des perturbations récurrentes liées soit aux coupes forestières, soit aux contraintes climatiques et à ses cascades de conséquences (mortalité accrue due aux sécheresses, tempêtes...). La composition des peuplements forestiers change donc continuellement. Pour paraphraser Héraclite: "On ne voit jamais deux fois le même peuplement forestier ", la structure et la composition sont une résultante de multiples interactions tant positives (facilitation) que négatives (compétition, parasitisme), qui certes s'affrontent de prime abord mais se concilient ensuite, sous les contraintes environnementales, pour renouveler sans cesse la composition des essences forestières et le cortège d'espèces que ces peuplements forestiers abritent.

Le changement est une condition intrinsèque de cet équilibre dynamique qui se dessine sous nos yeux. Ce n'est pas sans rappeler le bateau de Thésée dont les sophistes d'Athènes se demandaient, au fur et à mesure que les pièces en étaient modifiées ou remplacées pour cause d'usure, s'il s'agissait encore du même bateau avec sa charge symbolique c'est-à-dire le bateau qui a ramené Thésée, vainqueur du Minotaure. En tant que bateau, il remplirait toujours sa fonction de navigation, mais sa valeur symbolique aura-t-elle changé ou pas au fil des siècles?

Pour le massif forestier du Morvan, véritable mosaïque où s'imbriquent des peuplements exclusivement composés de feuillus et de résineux et des peuplements mixtes, est-ce toujours le même paysage forestier? Aurait-il perdu sa valeur symbolique? Assure-t-il toujours ses fonctions multiples?

Un paysage doit fonctionner dans ses composantes écologiques, socioéconomiques et culturelles. Si l'évolution de ses composantes s'opère de manière logique et 
compréhensible dans un certain "état du monde", alors l'acceptation de cette évolution est facile. Dans le Morvan, massif au paysage forestier feuillu, le sens commun veut que l'on y voie des hêtraies et des chênaies-hêtraies parcourues par des coupes d'amélioration et de régénération. Si de vastes coupes rases sur des versants entiers suivies de reboisement en résineux remplacent les feuillus, la logique du paysage est rompue. Par exemple, le diagnostic paysager conduit en 2017 au sein du Grand Site de France mont Beuvray-Bibracte explicite ce qui signe le cachet des paysages du Beuvray :

"Le dégagement des pâtures et des chemins dans les vallons (leur "visibilité") souligne, par contraste, le relief des monts boisés qui les dominent; le paysage est ainsi géographiquement compréhensible (sa "lisibilité")» et révèle les graves menaces qui pèsent sur les qualités paysagères et naturelles du Grand Site, notamment du fait de l'enfrichement des pâturages de pente et de fond de vallon (déprise agricole), de la mise à nu de pans de forêt (coupes rases), de la disparition de chemins (perte d'utilité)» (Goudiard, 2019).

Ce sentiment de rupture peut-il être surmonté en adoptant un autre point de vue, une autre approche?

\section{Penser comme une montagne ou la pensée chinoise du paysage}

Dans sa poétique du souffle du vide médian, François Cheng nous apprend que dès le ive siècle environ s'est élaborée, en Chine, une philosophie esthétique qui tentait de penser la beauté révélée par l'intime dialogue entre l'homme et la nature, et par les diverses formes de la création artistique (Cheng, 2004). Les maîtres ont dégagé un ensemble de réflexions qui se cristalliseront entre les xie et xiiie siècles autour de la notion centrale du quing-jiing, «sentiment paysage»: celui-ci désigne l'interpénétration de l'esprit humain et de l'esprit du monde, tous deux étant censés mus par le même qi, « souffle esprit », et par le même yi, « désir, élan, intentionnalité ».

41 Cette idée de "sentiment-paysage ", à son tour, connaîtra au cours des siècles un approfondissement continu. Le grand peintre Shitao du xviie siècle affirmait: «je détiens le nœud de la montagne, son cœur bat en moi » et Paul Cézanne, le plus proche peintre des grandes intuitions chinoises disait à propos de la Sainte-Victoire: «La montagne pense en moi, je deviens sa conscience. »

42 Cette philosophie orientale résonne curieusement avec celle d'autre-atlantique du «Penser comme une montagne » d'Aldo Leopold (1948) pour qui «le progrès ne vaut que si on arrive à faire éclore la réceptivité dans des cerveaux humains qui ne sont pas préparés et non à faire naître des routes dans des paysages merveilleux ».

43 Dans la pensée chinoise, il n'est jamais question de beauté, d'esthétisme dans le paysage car il resterait à sa surface. Qu'est ce qui valorise, en effet, un paysage? Guo Xi, lettré chinois du xie siècle le précise dans la typologie suivant :

- il y a ceux qu'on traverse ;

- ceux que l'on contemple de loin;

- ceux dans lesquels on se promène ;

- ceux que l'on habite.

Mais ce lettré chinois précise que tous ne se valent pas. Une hiérarchie de valeurs s'opère : c'est ceux qu'on habite ou dans lesquels on aime à séjourner, à se promener, 
où l'on se sent à l'aise qui sont les plus intensément vécus, prégnants. Ils sont devenus des milieux, on y est dans son élément.

Le concept de «vivre de paysage » de François Jullien (2014) propose donc que l'on s'y « nourrisse » : le propriétaire-gestionnaire gère, exploite et vend du bois, l'habitant $y$ trouve son cadre de vie et le touriste, le ressourcement nécessaire à sa trépidante vie urbaine. Dans ce qui fait "paysage», on devrait s'y libérer du «dégoût», de la lassitude, de la poussière et du vacarme du monde mondain ou urbain. Par les autoroutes interurbaines, le Morvan est ainsi à un peu plus d'une heure de Paris et à portée de week-end des régions peuplées allemandes, suisses, flamandes et néerlandaises (Cavailhès et Hilal, 2010).

\section{La qualité paysagère en question}

Les objectifs de qualité paysagère correspondent aux orientations que le territoire se fixe en matière de paysage. Ils correspondent à l'expression du projet. Il s'agit des grandes lignes qui permettront de guider l'évolution des paysages dans le sens souhaité et présideront à l'élaboration de projets stratégiques ou ponctuels, tout au long de la vie de la charte. Ces objectifs doivent être partagés sur le territoire et portés politiquement.

Les " objectifs de qualité paysagère ", tels que les énonce la Convention européenne du paysage (2000), désignent ainsi «la formulation par les autorités publiques compétentes, pour un paysage donné, des aspirations des populations en ce qui concerne les caractéristiques paysagères de leur cadre de vie ", c'est-à-dire l'objectif final que la société se fixe à elle-même en ce qui concerne ses paysages. Cette définition induit que :

- les objectifs de qualité paysagère sont formulés pour chacune des unités paysagères qui composent le territoire et se rapportent en particulier aux structures paysagères qui les caractérisent ;

- les objectifs de qualité paysagère sont formulés par les autorités publiques, après concertation avec les acteurs socioéconomiques notamment. Le terme de "population" comprend en effet les acteurs économiques, les représentants associatifs, les habitants, etc. L'enjeu est donc de prendre en compte les représentations sociales des paysages et en particulier de saisir les attentes ou les valeurs qui sont attachées aux paysages par les populations (valeur patrimoniale, valeur esthétique, valeur productive...). En outre, si les objectifs sont formulés par les autorités publiques, c'est parce qu'ils doivent permettre de répondre à l'intérêt général ;

- les objectifs de qualité paysagère peuvent relever de la protection, de la gestion et/ou de l'aménagement des paysages. En fonction des paysages considérés et des valeurs qui leur sont portées en particulier, ces objectifs ne visent pas que la protection, mais potentiellement la mise en valeur, la requalification (points noirs...). Le cahier des paysages $\mathrm{du}$ PNRM identifie 25 zones sensibles paysagères.

Comme l'énonce le préambule de la Convention européenne du paysage (2000), le paysage étant considéré comme un principe directeur pour l'amélioration de la qualité de vie des populations, et également comme une ressource favorable à l'activité économique, les acteurs économiques et les populations doivent participer, aux côtés des pouvoirs publics et des acteurs professionnels, à la définition et à la mise en œuvre de ces objectifs. 

paysagères sont recensées et 25 zones sensibles paysagères sont reportées au plan de parc. 15 mesures sont spécifiques à la gestion forestière. On retrouve classiquement la multifonctionnalité des forêts du Morvan, la prise en compte de trame de vieux bois, la promotion d'une sylviculture irrégulière, la préservation de la forêt feuillue, l'accroissement de la part des forêts publiques, l'accroissement de la mise en place de structures collectives de gestion durable, l'amélioration de la prise en compte des enjeux environnementaux et paysagers dans les documents d'urbanisme et de gestion forestière. Le Schéma de cohérence territoriale (SCOT) du pays Autunois-Morvan a notamment bien pris en compte la qualité des paysages forestiers dans sa future planification. Ces objectifs sont ambitieux mais nécessaires pour conserver, restaurer des paysages forestiers morvandiaux.

50 La puissance publique a des outils juridiques contraignants dont les sites classés pour préserver la qualité des paysages de certains territoires comme Vézelay, au nord, mont Beuvray-Bibracte-mont Preneley au centre et bientôt le massif d'Uchon au Sud. Toutefois, les autorités publiques ne peuvent faire du massif forestier du Morvan un vaste et unique site classé. Les 127 communes du PNRM pourront certes acquérir ou renforcer leur patrimoine forestier, mais elles ne pourront à elles seules acquérir l'ensemble du massif. Il faut composer avec ces 18000 propriétaires.

Figure 5. Peuplement de douglas en conversion en futaie irrégulière dans le massif du Haut-Folin (Morvan)

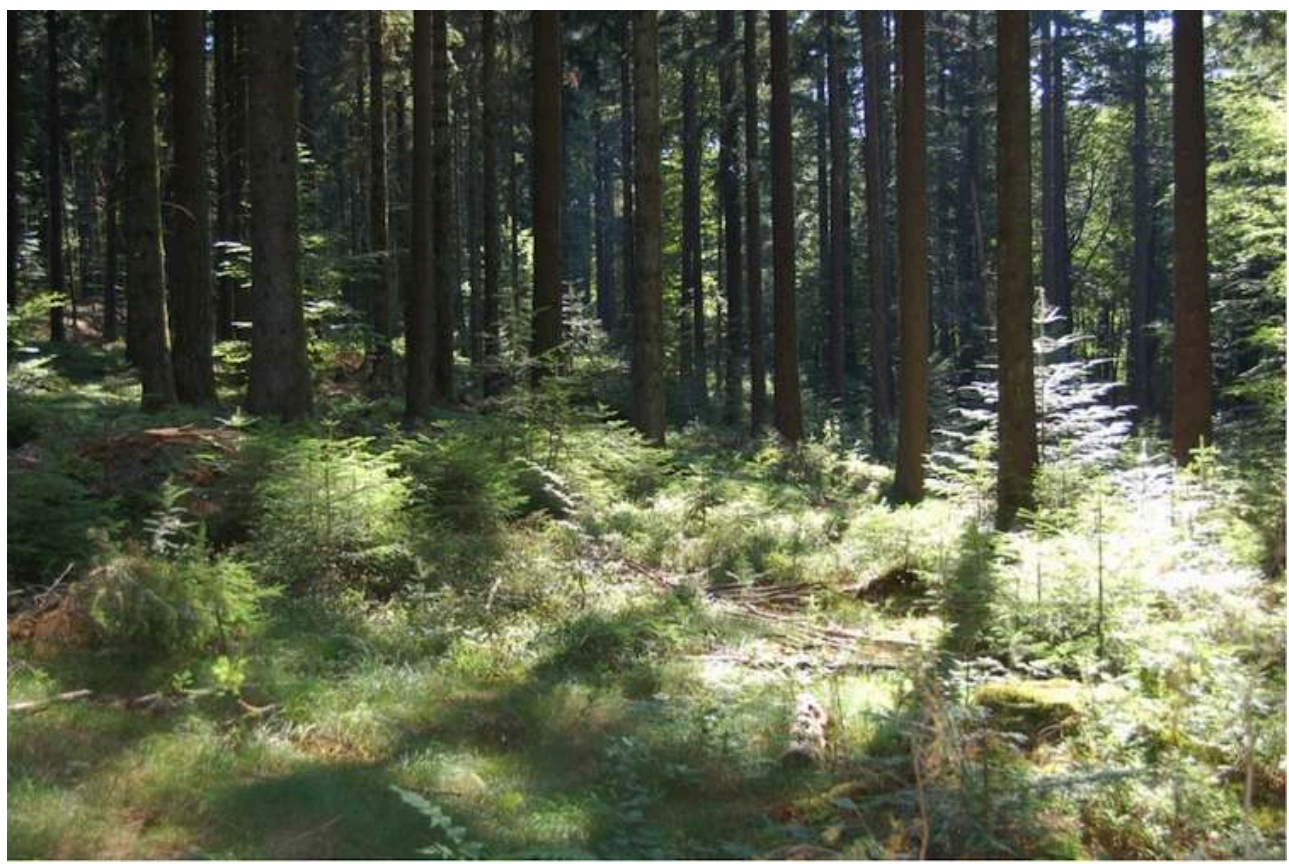

Dispositif Association Futaie irrégulière $n^{\circ} 16$.

Source : Association Futaie irrégulière.

51 S'il y a paysage, c'est qu'il y a échange entre ces entités. Il ne s'agit pas là d'agrément, d'ornements, de surplus, du fameux "supplément d'âme " car nous resterions alors coincés dans le paysage perçu et non vécu. Le paysage joue un rôle de compensation dans notre modernité: sa recherche est un remède à nos crises existentielles et écologiques. Pour Jullien (2014), c'est quand s'abolit « la coupure du tangible, physique,

Projets de paysage, 22 | 2020 
et du spirituel et que du spirituel se dégage à partir du physique» (ibid., p. 119). Un paysage vaut non pas uniquement par ses qualités définies mais par ce qui s'en diffuse, émane de lui.

C'est bien là un des premiers griefs des détracteurs d'une exploitation forestière qui fait perdre cette charge symbolique auprès du plus grand nombre : il n'émane plus grandchose d'un paysage forestier enrésiné puis exploité par coupe à blanc avec, de surcroît, des engins forestiers titanesques. Pour autant, une des conditions pour pouvoir, ensemble, vivre de paysage, c'est pour le forestier et l'écologue Aldo Leopold (1948) « ... un problème d'attitude et de mise en œuvre. Nous remodelons l'Alhambra à la pelleteuse, et nous sommes fiers de notre rendement. Nous n'allons pas abandonner la pelleteuse, qui, après tout, nous a rendu bien des services, mais nous avons besoin de critères d'une plus grande douceur et d'une plus grande objectivité pour l'utiliser avec succès ». En matière forestière, définir des objectifs de qualité paysagère ne suffit pas.

\section{Des enceintes de dialogue pour échanger, dialoguer et "vivre de paysage "}

53 Finalement, il apparaît essentiel que les propriétaires forestiers élargissent leurs regards pour considérer, au-delà de leurs seuls intérêts, qu'ils contribuent au bien commun d'un territoire, qu'ils en façonnent, comme les agriculteurs, l'image et les paysages et qu'ils portent une responsabilité importante dans sa "viabilité ». Ils y gagneraient reconnaissance et crédibilité, tout en préservant leur capital et ses revenus. Il convient pour cela d'observer et d'anticiper ce qui peut rééquiper le local face à cette économie mondialisée et de ne pas perdre de vue sa déterritorialisation : notre non-maitrise locale de ces enjeux économiques et politiques.

Autour de l'Établissement public de coopération culturelle (EPCC) de Bibracte s'est créé un collectif de chercheurs, structurant cette problématique. En s'appuyant sur des outils juridiques comme les associations syndicales foncières, les propriétaires forestiers de ce territoire, déjà très nombreux, le Parc du Morvan et le Centre archéologique, qui portent conjointement le site, préparent une stratégie paysagère pour le Grand Site Bibracte-mont Beuvray, qui est construit sur la nécessité d'« innover pour le paysage ». De ce travail il ressort que les décisions de politique publique ne touchent pas directement les paysages mais doivent procéder indirectement en visant les territoires et les acteurs, particulièrement dans les domaines de l'agriculture et de la forêt, qui " font le paysage "; ces décisions sont appelées en retour à se refléter dans le paysage. Le Grand Site est confronté à un problème classique de "verrouillage » de ses modèles agricoles, pastoraux et forestiers, que les agronomes qualifient de "dominants ", pour marquer le fait qu'ils découlent de l'hyperspécialisation et de la montée hégémonique de la monoculture: dans le Morvan, la production de veaux maigres charollais et de grumes de résineux douglas. C'est aux verrouillages de ces systèmes à bout de souffle qu'il faut s'attaquer aujourd'hui, via une dynamique renouvelée d'innovation. Un besoin d'innovation qui devient un enjeu majeur des politiques publiques et de leurs interactions avec la recherche. Depuis 2014, une coopération s'est installée entre des chercheurs du LabEx Item Innovation et territoires de montagne, l'équipe technique et les élus du Parc naturel régional du Morvan et de l'EPCC de Bibracte. Différentes arènes de débat autour du devenir du territoire et de son paysage se sont alors ouvertes, confrontant les intérêts des résidents permanents 
ou temporaires, des agriculteurs, des forestiers, des acteurs du tourisme, des gestionnaires de l'environnement. Une démarche anthropologique accompagne ce travail en considérant comment les habitants eux-mêmes vivent et voient la préservation de la ressource, comment ils s'impliquent positivement dans ce socioécosystème (Goudiard, 2019).

Leur attention se polarise toutefois sur des aspects souvent bien spécifiques, en rapport étroit avec leurs préoccupations ou leurs pratiques. En outre, ces affinités électives visà-vis du paysage relèvent pour une large part de la relation personnelle et du champ de l'intime, les personnes rencontrées confirmant avoir rarement eu l'occasion de les expliciter et de les évoquer avec d'autres. Revers de la médaille donc: comment, sur des bases aussi variables, voire insaisissables de prime abord, se repérer dans cette question de la qualité paysagère? Est-on inexorablement condamné au relativisme le plus total, chaque acteur voyant la question sous un angle qui lui est propre? (Bredif et al., 2017).

Il est donc crucial de définir, de partager les points de vue et de s'entendre sur ces fonctions multiples et sur les charges symboliques qui s'y rattachent. Pour Christian Barthod et Guy Landmann (2002) :

«Savoir partager ses émotions devant une belle forêt ou ce qui deviendra une belle forêt s'avère donc paradoxalement une condition de la crédibilité des forestiers dans leur participation à des débats de société. L'oublier laisse le champ libre à des discours irrationnels et séduisants qui opposent facilement la froideur rebutante du technicien et la chaleur sympathique du militant qui proclame son amour de la forêt. Mais, dans ce débat où l'esthétique a sa place, quels sont les moyens éthiquement acceptables pour les parties prenantes?»

Il faut travailler à un environnement de qualité ou qui le deviendra. C'est une réflexion à mener sur le champ des possibles, sur les fondements d'un sens commun à ce territoire forestier qu'est le Morvan : le sens est ce qui donne à la fois la direction de la mutation écologique, dans le registre de l'utile, la signification dans le registre du social et du politique et enfin la sensation, dans le registre du cadre et de la qualité de vie.

Nous tenons à exprimer nos remerciements à Vincent Guichard et Estelle Labbé-Bourdon pour la relecture du manuscrit et aux deux relecteurs pour leur contribution majeure à l'amélioration du manuscrit.

\section{BIBLIOGRAPHIE}

Arnould, P., « Discours sur le paysage : à la croisée des regards et des systèmes », Géoconfluences, 2003.

Barthod, C., et Landmann, G., « Pourquoi gérer la végétation forestière ? », Revue forestière française, t. LIV, no 6, 2002, p. 617-631.

Barthod, C., « Une approche globale dans notre monde de demain, importance sociale et sociétale, prise en compte de la biodiversité, multifonctionnalité, circuits courts », dans « La 
sylviculture à couvert continu ; un passé lointain, un présent certain, un futur serein », Pro Silva France, 2019, p. 1-12.

Barthod, C., « La multifonctionnalité des forêts entre discours et pratiques : illusion ou réalité à assumer ? ", Revue forestière française, t. LXVII, n 4, 2015, p. 293-319.

Boulard, D., « Capacité d'une chaîne de modélisation hydroclimatique haute résolution à simuler des indices de déficit hydrique : application aux douglasaies et hêtraies de Bourgogne ", thèse de géographie, université de Bourgogne, Dijon, 2016.

Bouleau, G., Deuffic, P., Sergent, A., Paillet, Y., et Gosselin, F., « Entre logique de production et de préservation : l'évolution de l'information environnementale dans les domaines de l'eau et de la forêt ", Vertigo. La revue électronique en sciences de l'environnement, vol. 16, $\mathrm{n}^{\circ}$ 2, septembre 2016, URL : http://journals.openedition.org/vertigo/17592; DOI : https://doi.org/10.4000/vertigo. 17592 .

Brédif, H., Simon, L. et Valenzisi, M., « Stakeholder motivation as a means toward a proactive shared approach to caring for biodiversity : Application on Plateau de Millevaches », Land Use Policy, vol. 61, 2017, p. 12-23.

Breman, P., « Cahier de recommandations à l'usage des sylviculteurs pour une approche paysagère de la production en forêt morvandelle », Dijon, préfecture de la région de Bourgogne, 1997, $104 \mathrm{p}$.

Cavailhès, J., et Hilal, M., « Dijon en 2030 : la ville resserrée ou étalée ? », Territoires 2040, n 22010 , p. 101-116.

Cheng, F., Le Livre du vide médian, Paris, Albin Michel, 2004.

Claudez, P., « Rapport d'audit concernant les conditions et les moyens d'une gestion forestière durable. Étude de cas : Le Parc naturel régional du Morvan », direction de la Nature et des Paysages, Fédération nationale des parcs, 2000.

Convention européenne du paysage, Conseil de l'Europe, 2000.

Corvol-Dessert, A., « La forêt. Les savoirs et le citoyen. Regards croisés sur les acteurs, les pratiques et les représentations » dans Vannier, P et Meiller, D. (dir.), La Forêt. Les savoirs et le citoyen, Chalon-sur-Saône, éditions ANCR, 1995.

Deconninck, M.-C., «L'état actuel de la forêt morvandelle ", Revue scientifique Bourgogne Nature, $\mathrm{n}^{\circ}$ 9, 2011, p. 136-139.

Dérioz, P., « La place du paysage dans l'action des parcs naturels régionaux depuis la loi paysage (1993) ", Projets de paysage, nº 9, décembre 2013, URL : http://www.projetsdepaysage.fr/fr/ la_place_du_paysage_dans_l_action_des_parcs_naturels_regionaux_depuis_la_loi_paysage_1993_

Déry, S., " Le paysage comme ressource », Vertigo. La revue électronique en sciences de l'environnement, février 2012, URL : http://journals.openedition.org/vertigo/11569; DOI : https:// doi.org/10.4000/vertigo.11569.

Domon, G., Tremblay, F., et Bélanger, L., « Le paysage comme composante incontournable de la gestion intégrée des ressources et des territoires », rapport final pour la convention d'étude sur la gestion de la forêt publique québécoise, chaire en paysage et environnement de l'université de Montréal, 2004.

Dupont, J., « Le flottage des bois du Morvan pour l'approvisionnement de Paris »., Les Annales des pays nivernais, vol. 76, 1995, p. 1-40. 
Fonds mondial pour la nature, « Restaurer la biodiversité des forêts », fiche 1, « Guide aprèstempête sur une gestion mélangée et irrégulière dans le Morvan et en Aquitaine », WWF, Sepanso, Autun Morvan Écologie, Pro-Silva, RNF, 2006.

Goudiard, R., «Vers le Morvan des solutions ? Trois approches par le terrestre, par le paysage, par le commun. Document de travail », EPCC Bibracte, 2019.

Haidara, S., Aury, N., Lardon, S., « Mobilisation supplémentaire de bois et acceptation sociale. Retour sur une expérience participative en Morvan », dans Jallais, A. et Morogues, F. de (coord.) « Entre dynamiques et mutations. Quelles voies pour la forêt et le bois ? ", Paris, GIP Ecofor, 2018, p. 54-57.

Hotyat, M., « Approche multidimensionnelle et cartographique du paysage forestier : recherche méthodologique appliquée au secteur de Dun-les-places », thèse de 3e cycle, université Paris 7Diderot, 1975.

Jullien, F., Vivre de paysage ou l'Impensé de la raison, Paris, Gallimard, 2014.

Leopold, A., Almanach d'un comté des sables suivi de quelques croquis (1948), Paris, Flammarion, 2000.

Marage, D., « La forêt dans tous ses états : de la conservation à l'évaluation territoriale », habilitation à diriger des recherches, université Paris 1 Panthéon Sorbonne, 2018.

Nemoz-Rajot, H., « Le régime forestier : une mosaïque moderne et évolutive », Revue forestière française, vol. 50, $\mathrm{n}^{\circ} 1,1998$, p. 10-19

Oberti, D., « Le choix des essences forestières dans le Morvan. Guide simplifié », Parc naturel régional du Morvan, 1997.

Ollagnon, H., « La gestion de la biodiversité : quelles stratégies patrimoniales ? », Annales des Mines, 2006. p. 50-57.

Ost, F., La Nature hors-la-loi : l'écologie à l'épreuve du droit, Paris, La Découverte, 1995.

Paris, A., « L'impact du flottage sur la société morvandelle », Revue scientifique Bourgogne Nature, vol. 9, 2011, p. 96-102.

Rameau, J., « L'intérêt chorologique de quelques groupements forestiers du Morvan, France », Plant Ecology, vol. 59, 1-3, 1985, p. 47-65.

Ranger, J., Augusto, L., Berthelot, A., Bouchon, J., Cacot, E., Dambrine, E., Gavaland, A., Laclau, J.P., Legout, A., Nicolas, M., « Sylviculture et protection des sols ", Revue forestière française, t. LXIII, $\mathrm{n}^{\circ} 2,2011$.

Riethmuller, T., Weiss, S., et Chauvin, C., « Les chartes forestières de territoire : premier bilan », Ingénieries-Eaux Aménagements Territoire, $\mathrm{n}^{\circ}$ 36, 2003, p. 43.

Rivière, C., « De la ressource "bois" à la forêt multifonctionnelle ? Étude du plan simple de gestion forestière au sein du PNR des Alpilles ", Développement durable et territoires, vol. 8, n 1, 2017.

Ruch, P., et Montagny, X., « Le parc de machines d'exploitation forestière en bourgogne en 20 $14 »$, FCBA, 2015.

Saint-Jacob, P. de, Les Paysans de la Bourgogne du Nord au dernier siècle de l'Ancien régime, Bernigaud et Privat, 1960.

Sansilvestri, R., Cuccarollo, M., Frascaria-Lacoste, N., Benito-Garzon, M., Fernandez-Manjarres, J., «Evaluating climate change adaptation pathways through capital assessment : five case studies of forest social-ecological systems in France ", Sustainability Science, vol. 15, n 2, March 2020, p. 539-553. 
Simonnot, J.-L., « Contribution à la connaissance des rapports sol-végétation en forêt du Morvan », thèse de doctorat, université Henri Poincaré-Nancy 1, 1991.

\section{RÉSUMÉS}

Le paysage est devenu le fil rouge de la charte du Parc naturel régional du Morvan 2020-2035. Les parties prenantes ont établi le double constat que considérer l'avenir d'un paysage forestier oblige à prendre en compte de façon systémique les activités qui se déploient sur ce territoire et surtout que le paysage peut être un excellent levier de motivation et de médiation entre acteurs. Par un traitement des attentes culturelles ou sociétales, l'enrichissement et la validation du projet par la population sont appelés à faire vivre le paysage. Un paysage doit fonctionner dans ses composantes écologiques, socioéconomiques et culturelles. Si l'évolution de ses composantes s'opère de manière logique et compréhensible dans un certain "état du monde", alors l'acceptation de cette évolution est facile. Le paysage ne peut plus être « la partie d'un pays que la nature présente à un observateur ». Dans ce découpage objet-sujet, les paysages forestiers du Morvan nous offrent parfois un spectacle de désolation, de vide. Ce couplage sujet-objet propre à la définition occidentale du paysage pourrait laisser place à une vision issue de la pensée chinoise. Nous verrons comment la production de catégories émergentes à partir d'enceintes de concertation territorialisée permet de faire travailler ensemble les acteurs autour de ce qui peut être préservé en commun, le vivre de paysage. L'issue à ces antagonismes se trouve dans la construction d'une vision partagée de l'avenir du paysage du massif. Nous étayerons nos propos grâce aux nombreux bilans et enquêtes réalisées lors de la révision de la charte.

The landscape has become the red thread of the 2020-2035 Charter for the Morvan Regional Nature Park. Stakeholders realise that in considering the future of a forest landscape they need to systemically take into account the activities deployed within it and, above all, to recognise the landscape as an excellent lever for motivation and mediation between actors. By addressing cultural or societal expectations, enhancing the project, and gaining the population's approval can ensure the landscape is experienced as a living space. A landscape must function in relation to its ecological, socio-economic, and cultural components. If these components evolve in a logical and comprehensible way in tune with a certain "state of the world", their change is easily accepted. The landscape can therefore no longer be perceived as "the part of a region nature presents to an observer". From this object-subject perspective, the forest landscapes of the Morvan sometimes offer us a spectacle of desolation and emptiness. This coupling of subject and object which is specific to the Western definition of the landscape could be replaced by a vision derived from Chinese thought. We will see how the production of emerging categories based on regional consultation procedures can enable actors to work together on preserving the shared experience of the landscape. The solution to such oppositions lies in the construction of a shared vision of the future of the landscape of the Morvan Massif. We will back up the arguments put forward in this article with the many evaluations and surveys carried out during the revision of the charter.

\section{INDEX}

Mots-clés : gouvernance, conflits d'usages, valeurs, forêt tempérée, aire protégée

Keywords : governance, conflicts of use, values, temperate forest, protected area 


\section{AUTEUR}

\section{DAMIEN MARAGE}

Après avoir été maître de conférences à AgroParisTech-Engref en écologie forestière, Damien

Marage est actuellement responsable du pôle Territoires à la Dreal Bourgogne-Franche-Comté.

Spécialiste de la gestion des espaces naturels, il est titulaire d'une HDR en géographie et chercheur associé au Ladyss CNRS UMR 7533 CNRS.

marage.damien[at]gmail[dot]com 